\title{
Canal Surface Whose Center Curve is a Hyperbolic Curve with Hyperbolic Frame
}

\author{
Ali Uçum* \\ (Dedicated to the memory of Prof. Dr. Aurel BEJANCU (1946 - 2020))
}

\begin{abstract}
In this paper, we obtain the parametrization of the canal surfaces whose center curves are the hyperbolic curves on the hyperbolic space $H^{2}$ in $\mathbb{E}_{1}^{3}$. The parametrization of the canal surface is expressed according to the hyperbolic frame given in [10]. Then, the parallel surface of this surface is studied. Also, we define the notion of the associated canal surface. Lastly, we give the geometric properties of these surfaces such that Weingarten surface, $(X, Y)$-Weingarten surface and linear Weingarten surface.
\end{abstract}

Keywords: Canal surfaces, tubular surfaces, hyperbolic curve, Weingarten surface, $(X, Y)$-Weingarten surface.

AMS Subject Classification (2020): Primary: 53A35 ; Secondary: 53B30; 53C50.

\section{Introduction}

Canal surfaces was firstly investigated by Monge in 1850. A canal surface is defined as a surface formed as the envelope of a family of spheres whose centers lie on a space curve $C(t)$ with radius $r(t)$. If the radius $r(t)$ is constant, then the canal surface is called as pipe surface or tubular surface. Canal surfaces play an essential role in descriptive geometry, because in case of an orthographic projection its contour curve can be drawn as the envelope of circles. In technical area canal surfaces can be used for blending surfaces smoothly. Canal surface is useful to represent various objects e.g. pipe, hose, rope or intestine of a body. Moreover, canal surface is an important instrument in surface modelling for CAD/CAM such as tubular surfaces, torus and Dupin cyclides [7].

Canal surfaces and tubular surfaces have been studied by many researchers. In [5], [6], [7], [8], the authors study canal surfaces and tubular surfaces in Euclidean 3-space, Minkowski 3-space, Galilean and Pseudo Galilean spaces. Lately, in [13], the authors consider the new approach to canal surfaces. Also in [2] and [9], the authors study canal surfaces with quaternions.

In [10], the author defines the hyperbolic frame of the curves on the sphere $H^{2}$. Also the curves with constant curvatures were studied in many papers such as [3],[4] and [11].

In this paper, we obtain the parametrization of the canal surfaces whose center curves are the hyperbolic curves on the hyperbolic space $H^{2}$ in $\mathbb{E}_{1}^{3}$. The parametrization of the canal surface is expressed according to the hyperbolic frame given in [10]. Then the parallel surface of this surface is studied. Also we define the notion of the associated canal surface. Lastly we give the geometric properties of these surfaces such that Weingarten surface, $(X, Y)$-Weingarten surface and linear Weingarten surface.

\section{Preliminaries}

The Minkowski space $\mathbb{E}_{1}^{3}$ is the Euclidean 3-space $\mathbb{E}^{3}$ equipped with indefinite flat metric given by

$$
d s^{2}=-d x_{1}^{2}+d x_{2}^{2}+d x_{3}^{2},
$$


where $\left(x_{1}, x_{2}, x_{3}\right)$ is a rectangular coordinate system of $\mathbb{E}_{1}^{3}$. We denote by $\langle$,$\rangle the inner product on \mathbb{E}_{1}^{3}$.

Let $m$ be a fixed point and $r>0$ be a constant. The pseudo-Riemannian sphere is defined by

$$
S_{1}^{2}(m, r)=\left\{u \in \mathbb{E}_{1}^{3}:\langle u-m, u-m\rangle=r^{2}\right\} ;
$$

the pseudo-Riemannian hyperbolic space is defined by

$$
H^{2}(m, r)=\left\{u \in \mathbb{E}_{1}^{3}:\langle u-m, u-m\rangle=-r^{2}\right\} ;
$$

the pseudo-Riemannian lightlike cone is defined by

$$
Q^{2}(m)=\left\{u \in \mathbb{E}_{1}^{3}:\langle u-m, u-m\rangle=0\right\} .
$$

Now, for a unit speed regular curve $x(s) \subset H^{2} \subset \mathbb{E}_{1}^{3}$, we choose $\{x(s), \alpha(s), y(s)\}$ forming a standart Pseudo orthonormal basis of $\mathbb{E}_{1}^{3}$. Then the hyperbolic Frenet formulas of the hyperbolic curve $x(s)$ in $H^{2}$ can be written as

$$
x^{\prime}(s)=\alpha(s), \quad \alpha^{\prime}(s)=x(s)+\kappa(s) y(s), \quad y^{\prime}(s)=-\kappa(s) \alpha(s)
$$

where $\langle x(s), x(s)\rangle=-1$ and $\langle\alpha(s), \alpha(s)\rangle=\langle y(s), y(s)\rangle=1$.

Here, the function $\kappa(s)$ is called the hyperbolic curvature function (or curvature) of $x(s)$ and the frame $\{x(s), \alpha(s), y(s)\}$ is called the hyperbolic Frenet frame of the hyperbolic curve $x(s)$ ([10]).

We recall some well-known formulas for the surfaces in $\mathbb{E}_{1}^{3}$. Let $M$ be a surface of $\mathbb{E}_{1}^{3}$, the standart connection $D$ on $\mathbb{E}_{1}^{3}$ induces the Levi-Civita connection $\nabla$ on $M$. We have the following Gauss formula

$$
D_{X} Y=\nabla_{X} Y+h(X, Y),
$$

and the Weingarten formula

$$
D_{X} \xi=-A_{\xi} X+{ }^{\perp} \nabla_{X} \xi,
$$

where $X, Y \in \Gamma(T M)$ and $\xi \in \Gamma\left(T M^{\perp}\right)$. Then $\nabla$ is the Levi-Civita connection of $M, h$ is the second fundamental form, $A_{\xi}$ is the shape operator, and ${ }^{\perp} \nabla$ is the normal connection. We note that

$$
\langle h(X, Y), \xi\rangle=\left\langle A_{\xi} X, Y\right\rangle .
$$

The mean curvature vector field $\vec{H}$, the mean curvature $H$ and the Gauss curvature of $M$ are given respectively by

$$
\vec{H}=\frac{1}{2}\left(h\left(e_{1}, e_{1}\right)+h\left(e_{2}, e_{2}\right)\right), \quad H=\|\vec{H}\| \quad \text { and } \quad K=\operatorname{det} A
$$

where $\left\{e_{1}, e_{2}\right\}$ is an pseudo orthonormal basis on $M([1])$.

Let $U$ be the unit normal vector field on a surface $M(s, t)$ defined by

$$
U=\frac{M_{s} \times M_{t}}{\left\|M_{s} \times M_{t}\right\|} .
$$

The second fundamental form $I I$ of a surface $M(s, t)$ is given as

$$
I I=e d s^{2}+2 f d s d t+g d t^{2}
$$

where

$$
e=g\left(M_{s s}, U\right), f=g\left(M_{s t}, U\right), g=g\left(M_{t t}, U\right) .
$$

([14]) Thus the second Gaussian curvature $K_{I I}$ of a surface is given as

$$
\begin{aligned}
& K_{I I}=\frac{1}{\left(e g-f^{2}\right)^{2}}\left\{\begin{array}{ccc}
-\frac{1}{2} e_{t t}+f_{s t}-\frac{1}{2} g_{s s} & \frac{1}{2} e_{s} & f_{s}-\frac{1}{2} e_{t} \\
f_{t}-\frac{1}{2} g_{s} & e & f \\
\frac{1}{2} g_{t} & f & g
\end{array} \mid\right. \\
& \left.-\left|\begin{array}{ccc}
0 & \frac{1}{2} e_{t} & \frac{1}{2} g_{s} \\
\frac{1}{2} e_{t} & e & f \\
\frac{1}{2} g_{s} & f & g
\end{array}\right|\right\}
\end{aligned}
$$




\section{Canal surfaces with the hyperbolic curve in $H^{2}$}

In this section, we consider the canal surfaces whose center curve is the hyperbolic curves in $H^{2}$. The following theorem can be obtained by similar technique in [13]. Thus we omit the proof.

Theorem 3.1. Let $x(s)$ be a hyperbolic curve with arc-length parameter $s$ on $H^{2}$ and be the center curve of a canal surface

(i) obtained from the sphere $S^{2}(r)$. Then the parametrization of the canal surface can be as following

$$
\begin{aligned}
M(s, t)= & \left(1+m_{1} r(s) \sqrt{1-r_{s}^{2}(s)} \sinh t\right) x(s)-r(s) r_{s}(s) \alpha(s) \\
& +\left(m_{2} r(s) \sqrt{1-r_{s}^{2}(s)} \cosh t\right) y(s)
\end{aligned}
$$

and the parametrization of the tubular surface can be as following

$$
M(s, t)=\left(1+m_{1} r \sinh t\right) x(s)+\left(m_{2} r \cosh t\right) y(s)
$$

(ii) obtained from the hyperbolic space $H^{2}(r)$. Then the parametrization of the canal surface can be as following

$$
\begin{aligned}
M(s, t)= & \left(1+m_{1} r(s) \sqrt{1+r_{s}^{2}(s)} \cosh t\right) x(s)+r(s) r_{s}(s) \alpha(s) \\
& +\left(m_{2} r(s) \sqrt{1+r_{s}^{2}(s)} \sinh t\right) y(s)
\end{aligned}
$$

and the parametrization of the tubular surface can be as following

$$
M(s, t)=\left(1+m_{1} r \cosh t\right) x(s)+\left(m_{2} r \sinh t\right) y(s)
$$

(iii) obtained from the ligthlike cone $Q^{2}$. Then the parametrization of the canal surface(tubular surface) can be as following

$$
M(s, t)=(1+a(s, t)) x(s)+m_{1} a(s, t) y(s)
$$

where $m_{1}, m_{2} \in\{-1,1\}$ and $a(s, t)$ is a function of $s$ and $t$, but not only $s$.

Remark 3.1. It is clear that the surface

$$
M(s, t)=(1+a(s, t)) x(s)+m_{1} a(s, t) y(s)
$$

is a degenerate surface.

In the following theorem, we classify all hyperbolic curve on $H^{2}$ with constant curvature.

Theorem 3.2. Let $\kappa$ be a real number. Then $x(s)$ is a hyperbolic curve on $H^{2}$ with arc-length parameter s and curvature $\kappa$ satisfying

(i) $\kappa^{2}<1$ if and only if $x(s)$ can be parameterized by

$$
x=\cosh \left(\sqrt{1-\kappa^{2}} s\right) V_{1}+\sinh \left(\sqrt{1-\kappa^{2}} s\right) V_{2}+V_{3}
$$

where $V_{1}, V_{2}, V_{3}$ are mutually orthogonal vectors satisfying the following equations

$$
\left\langle V_{1}, V_{1}\right\rangle=-\left\langle V_{2}, V_{2}\right\rangle=-\frac{1}{1-\kappa^{2}} \quad \text { and } \quad\left\langle V_{3}, V_{3}\right\rangle=\frac{\kappa^{2}}{1-\kappa^{2}} .
$$

(ii) $\kappa^{2}>1$ if and only if $x(s)$ can be parameterized by

$$
x=\cos \left(\sqrt{\kappa^{2}-1} s\right) V_{1}+\sin \left(\sqrt{\kappa^{2}-1} s\right) V_{2}+V_{3}
$$

where $V_{1}, V_{2}, V_{3}$ are mutually orthogonal vectors satisfying the following equations

$$
\left\langle V_{1}, V_{1}\right\rangle=\left\langle V_{2}, V_{2}\right\rangle=\frac{1}{\kappa^{2}-1} \quad \text { and } \quad\left\langle V_{3}, V_{3}\right\rangle=-\frac{\kappa^{2}}{\kappa^{2}-1} .
$$


(iii) $\kappa^{2}=1$ if and only if $x$ ( $s$ can be parameterized by

$$
x=s^{2} V_{1}+s V_{2}+V_{3}
$$

where

$$
\begin{gathered}
\left\langle V_{1}, V_{1}\right\rangle=\left\langle V_{1}, V_{2}\right\rangle=\left\langle V_{2}, V_{3}\right\rangle=0, \\
\left\langle V_{2}, V_{2}\right\rangle=-\left\langle V_{3}, V_{3}\right\rangle=1 \quad \text { and }\left\langle V_{1}, V_{3}\right\rangle=-\frac{1}{2} .
\end{gathered}
$$

Proof. Let $x(s)$ be a hyperbolic curve on $H^{2}$ with arc-length parameter $s$ and constant curvature $\kappa$. By using the hyperbolic Frenet equations (4.2), we obtain the following homogeneous differential equation with constant coefficients

$$
x^{\prime \prime \prime}+\left(\kappa^{2}-1\right) x^{\prime}=0 .
$$

The characteristic equation of the previous equation is follows

$$
r\left(r^{2}+\left(\kappa^{2}-1\right)\right)=0 .
$$

(i) Assume that $\kappa^{2}<1$. Then we get

$$
x=\cosh \left(\sqrt{1-\kappa^{2}} s\right) V_{1}+\sinh \left(\sqrt{1-\kappa^{2}} s\right) V_{2}+V_{3} .
$$

Differentiating (3.1) with respect to $s$, we get

$$
\alpha=\sqrt{1-\kappa^{2}} \sinh \left(\sqrt{1-\kappa^{2}} s\right) V_{1}+\sqrt{1-\kappa^{2}} \cosh \left(\sqrt{1-\kappa^{2}} s\right) V_{2} .
$$

By using $\langle\alpha, \alpha\rangle=1$, we get $V_{1}, V_{2}, V_{3}$ are mutually orthogonal vectors satisfying the following equations

$$
\left\langle V_{1}, V_{1}\right\rangle=-\left\langle V_{2}, V_{2}\right\rangle=-\frac{1}{1-\kappa^{2}} \quad \text { and } \quad\left\langle V_{3}, V_{3}\right\rangle=\frac{\kappa^{2}}{1-\kappa^{2}} .
$$

(ii) Assume that $\kappa^{2}>1$. Then the proof is similar.

(iii) Assume that $\kappa^{2}=1$. Then we get

$$
x=s^{2} V_{1}+s V_{2}+V_{3} .
$$

Differentiating (3.2) with respect to $s$, we get

$$
\alpha=2 s V_{1}+V_{2}
$$

By using $\langle x, x\rangle=-1$ and $\langle\alpha, \alpha\rangle=1$, we get $\left\langle V_{1}, V_{1}\right\rangle=\left\langle V_{1}, V_{2}\right\rangle=\left\langle V_{2}, V_{3}\right\rangle=0$,

$$
\left\langle V_{2}, V_{2}\right\rangle=-\left\langle V_{3}, V_{3}\right\rangle=1 \quad \text { and } \quad\left\langle V_{1}, V_{3}\right\rangle=-\frac{1}{2} .
$$

Then the proof is complete.

Example 3.1. Let us take $\kappa=\frac{\sqrt{2}}{2}$ in $(i)$ of Theorem 3.2. Then we obtain

$$
\left\langle V_{1}, V_{1}\right\rangle=-\left\langle V_{2}, V_{2}\right\rangle=-2 \text { and }\left\langle V_{3}, V_{3}\right\rangle=1 .
$$

Then we can choose

$$
V_{1}=(\sqrt{2}, 0,0), \quad V_{2}=(0, \sqrt{2}, 0), \quad V_{3}=(0,0,1)
$$

which implies that

$$
\begin{aligned}
& x=\left(\sqrt{2} \cosh \left(\frac{s}{\sqrt{2}}\right), \sqrt{2} \sinh \left(\frac{s}{\sqrt{2}}\right), 1\right), \\
& \alpha=\left(\sinh \left(\frac{s}{\sqrt{2}}\right), \cosh \left(\frac{s}{\sqrt{2}}\right), 0\right), \\
& y=\left(-\cosh \left(\frac{s}{\sqrt{2}}\right),-\sinh \left(\frac{s}{\sqrt{2}}\right),-\sqrt{2}\right) .
\end{aligned}
$$

Now let us take $m_{1}=m_{2}=1$ in Theorem 3.1 and give the canal surfaces in Figure 2 . 

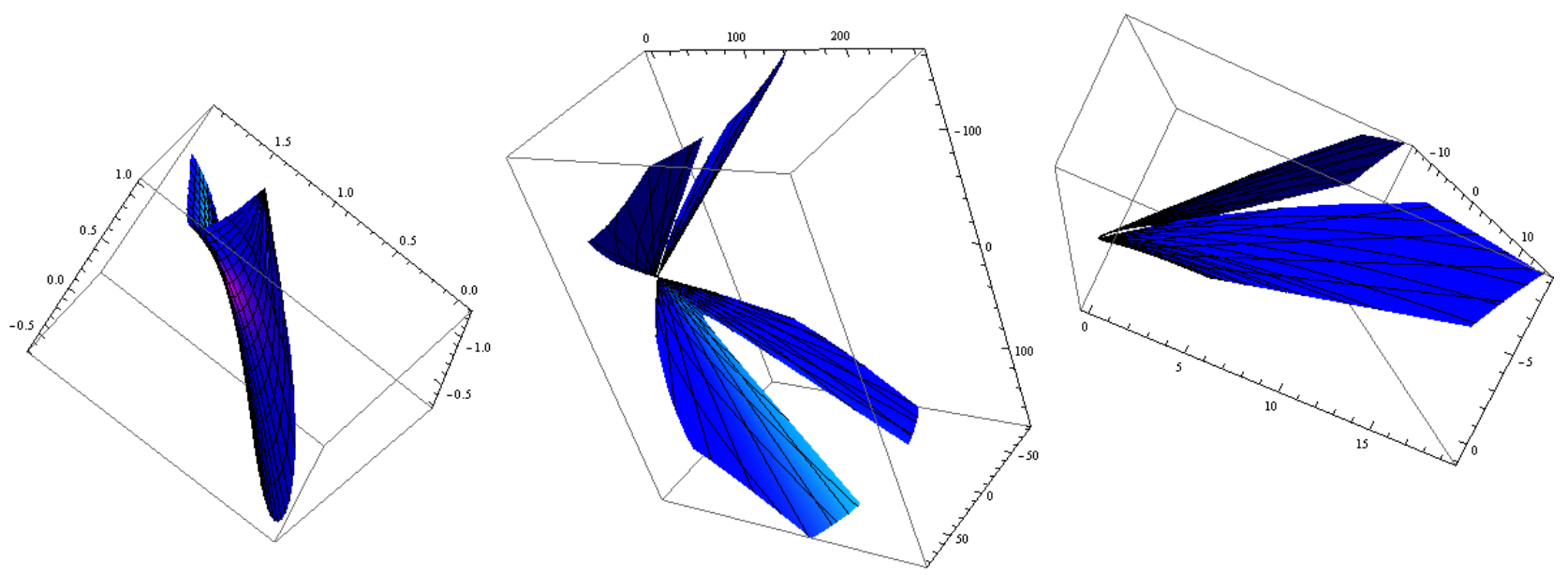

Figure 1. The canal surface for $r=e^{s}$ in (i) of Theorem 3.1 (left), $r=s$ in (ii) of Theorem 3.1(middle) and $a=s^{2} \cosh t$ in (iii) of Theorem 3.1(right).

Example 3.2. Let us take $\kappa=\sqrt{2}$ in $(i i)$ of Theorem 3.2. Then we choose

$$
V_{1}=(0,1,0), \quad V_{2}=(0,0,1), \quad V_{3}=(\sqrt{2}, 0,0)
$$

which implies that

$$
\begin{aligned}
& x=(\sqrt{2}, \cos s, \sin s), \\
& \alpha=(0,-\sin s, \cos s), \\
& y=(-1,-\sqrt{2} \cos s,-\sqrt{2} \sin s) .
\end{aligned}
$$

Now let us take $m_{1}=m_{2}=1$ in Theorem 3.1 and give the canal surfaces in Figure 3 .
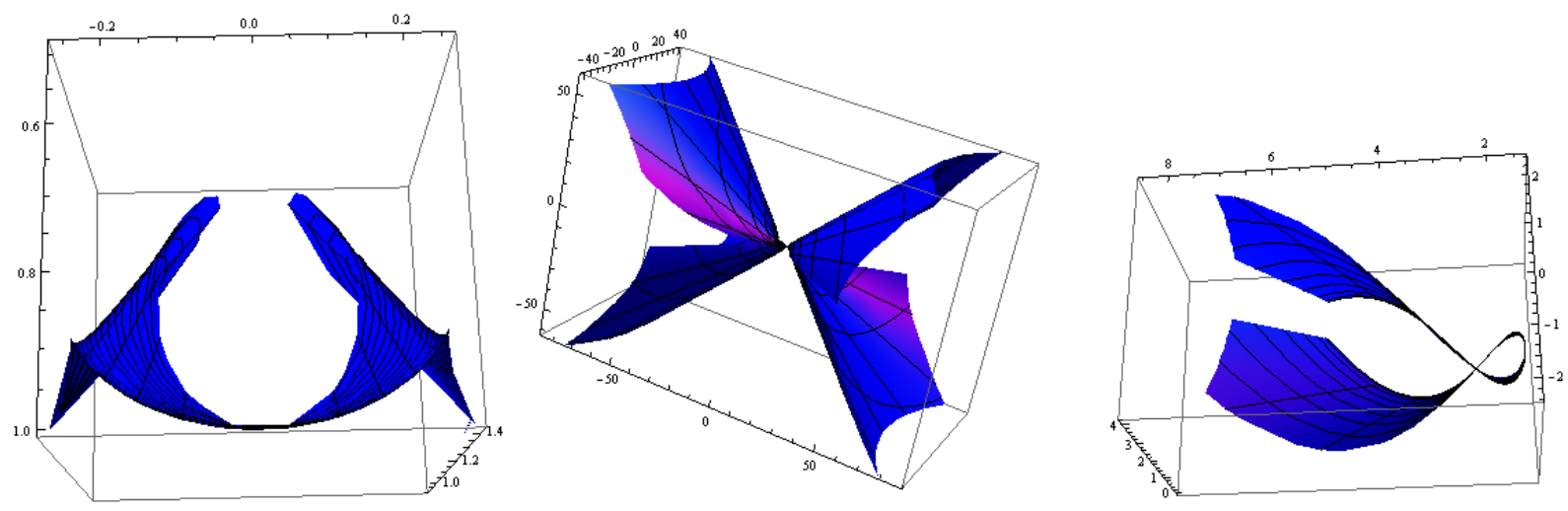

Figure 2. The canal surface for $r=s^{2}$ in (i) of Theorem 3.1 (left), $r=s$ in (ii) of Theorem 3.1(middle) and $a=s^{2} \cosh t$ in (iii) of Theorem 3.1(right).

Example 3.3. Let us take $\kappa=1$ in $(i i)$ of Theorem 3.2. Then we choose

$$
V_{1}=\left(\frac{1}{2}, 0, \frac{1}{2}\right), \quad V_{2}=(0,1,0), \quad V_{3}=(1,0,0)
$$


which implies that

$$
\begin{aligned}
x & =\left(\frac{s^{2}}{2}+1, s, \frac{s^{2}}{2}\right), \\
\alpha & =(s, 1, s), \\
y & =\left(-\frac{s^{2}}{2},-s, 1-\frac{s^{2}}{2}\right) .
\end{aligned}
$$

Now let us take $m_{1}=m_{2}=1$ in Theorem 3.1 and give the canal surfaces in Figure 4 .
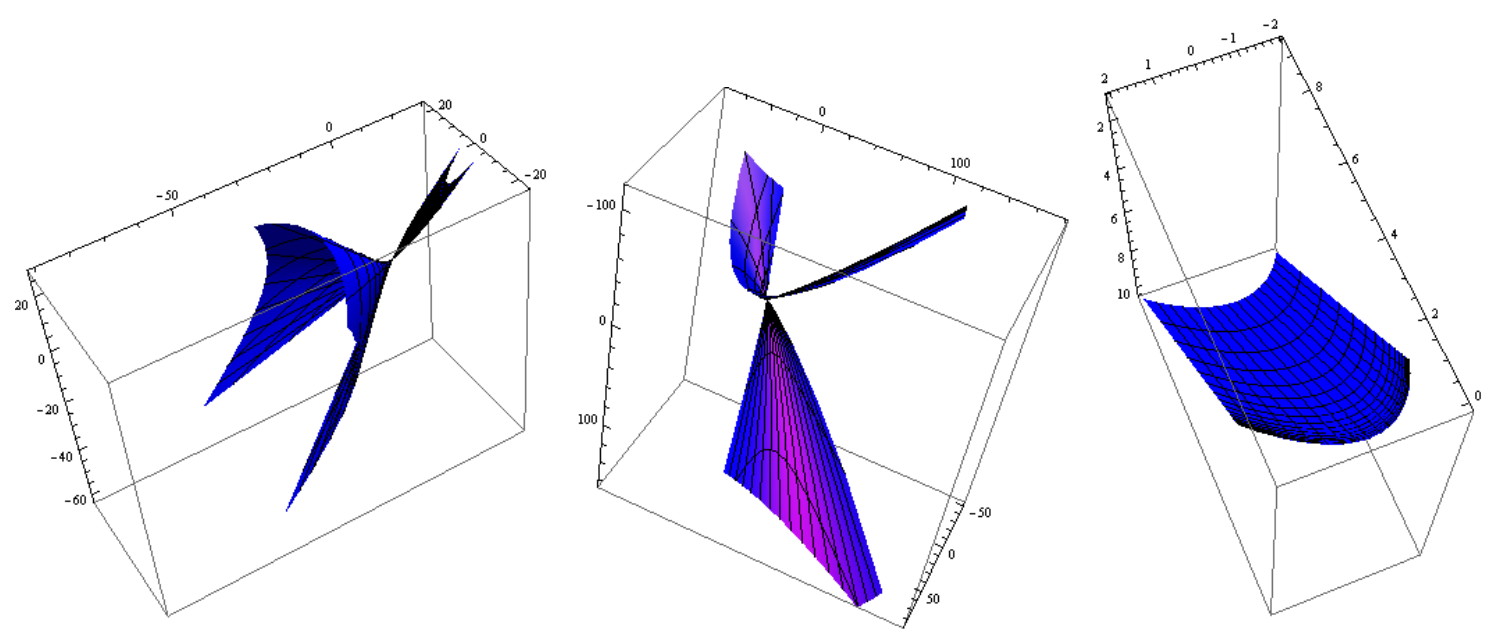

Figure 3. The canal surface for $r=2$ in (i) of Theorem 3.1 (left), $r=s^{3}$ in (ii) of Theorem 3.1(middle) and $a=e^{t}$ in (iii) of Theorem 3.1(right).

\section{Tubular surface whose center curve is the hyperbolic curve}

In this section we consider the tubular surface whose center curve is the hyperbolic curve in $H^{2}$.

\subsection{Tubular surface obtained from the sphere $S_{1}^{2}(r)$}

In this subsection, we consider the tubular surface obtained from the sphere $S_{1}^{2}(r)$, given in theorem 3.1. We assume that $m_{1}=m_{2}=1$. So we give the parametrization of the tubular surface as

$$
\psi(s, t)=(1+r \sinh t) x+(r \cosh t) y(s)
$$

From (4.1), we find

$$
\begin{aligned}
& \psi_{s}=(1+r \sinh t-r \kappa \cosh t) \alpha, \\
& \psi_{t}=(r \cosh t) x-(r \sinh t) y .
\end{aligned}
$$

We can find the components of first fundemental form as follows

$$
g_{11}=\left\langle\psi_{s}, \psi_{s}\right\rangle=(1+r \sinh t-r \kappa \cosh t)^{2}, \quad g_{12}=\left\langle\psi_{s}, \psi_{t}\right\rangle=0, \quad g_{22}=\left\langle\psi_{t}, \psi_{t}\right\rangle=-r^{2} .
$$

Then $g_{11} g_{22}-\left(g_{12}\right)^{2}=r^{2}(1+r \sinh t-r \kappa \cosh t)^{2}$. We assume that $1+r \sinh t-r \kappa \cosh t>0$ for the regularity of the surface $\psi$.

Now we will give an orthonormal basis on $\psi(s, t)$.

$$
\begin{aligned}
& e_{1}=\frac{1}{\left\|\psi_{s}\right\|} \psi_{s}=\alpha, \\
& e_{2}=\frac{1}{\left\|\psi_{t}\right\|} \psi_{t}=(\cosh t) x-(\sinh t) y
\end{aligned}
$$


where $\left\{e_{1}, e_{2}\right\}$ is an orthonormal frame field on $\psi(s, t)$ with signature $(+,-)$. Set

$$
e_{3}=-(\sinh t) x-(\cosh t) y
$$

where $e_{3}$ is a normal vector field to $\psi(s, t) .\left\{e_{1}, e_{2}, e_{3}\right\}$ is an orthonormal basis on $\psi(s, t)$. Then we obtain

$$
\begin{aligned}
D_{e_{1}} e_{1} & =\frac{1}{1+r \sinh t-r \kappa \cosh t}(x+\kappa y), \\
D_{e_{1}} e_{2} & =\frac{\cosh t-\kappa \sinh t}{1+r \sinh t-r \kappa \cosh t} \alpha, \\
D_{e_{2}} e_{2} & =\frac{1}{r}((\sinh t) x+(\cosh t) y) .
\end{aligned}
$$

The components of the second fundamental form $h$ are calculated as follows

$$
\begin{aligned}
& h_{11}=\left\langle D_{e_{1}} e_{1}, e_{3}\right\rangle=\frac{\sinh t-\kappa \cosh t}{1+r \sinh t-r \kappa \cosh t}, \\
& h_{12}=\left\langle D_{e_{1}} e_{2}, e_{3}\right\rangle=0 \text { and } h_{22}=\left\langle D_{e_{2}} e_{2}, e_{3}\right\rangle=-\frac{1}{r} .
\end{aligned}
$$

Theorem 4.1. The mean curvature $H$ of $\psi(s, t)$ is obtained as

$$
H=\frac{1}{2}\left(h_{11}-h_{22}\right)=\frac{1-2 r \kappa \cosh t+2 r \sinh t}{2 r(1+r \sinh t-r \kappa \cosh t)} .
$$

Theorem 4.2. The Gauss curvature $K$ of $\psi(s, t)$ is obtained as

$$
K=-\left(h_{11} h_{22}-\left(h_{12}\right)^{2}\right)=\frac{\sinh t-\kappa \cosh t}{r(1+r \sinh t-r \kappa \cosh t)} .
$$

A surface is called Weingarten surface if there exist a non-trivial function $\Psi(K, H)$ such that $\Psi(K, H)=$ $K_{s} H_{t}-K_{t} H_{s}=0$ for the Gauss curvature $K$ and mean curvature $H$ of the surface. Here subscripts denote partial derivatives. Also we a surface is called as a linear Weingarten surface if there exist real numbers $a$, $b, c \in \mathbb{R} \backslash\{0\}$ such that the linear combination $a K+b H=c$ is satisfied. For $(X, Y) \in\left\{\left(K, K_{I I}\right),\left(H, K_{I I}\right)\right\}$, the surface is called as $(X, Y)$-Weingarten surface if $\Psi(X, Y)=0$ ([12]).

From (4.2) and (4.3), we have

$$
K_{s}=\frac{-\kappa^{\prime} \cosh t}{r(1+r \sinh t-r \kappa \cosh t)^{2}}, \quad K_{t}=\frac{\cosh t-\kappa \sinh t}{r(1+r \sinh t-r \kappa \cosh t)^{2}}
$$

and

$$
H_{s}=\frac{-\kappa^{\prime} \cosh t}{2(1+r \sinh t-r \kappa \cosh t)^{2}}, \quad H_{t}=\frac{\cosh t-\kappa \sinh t}{2(1+r \sinh t-r \kappa \cosh t)^{2}} .
$$

Thus it can be easily seen that $\Psi(K, H)=K_{s} H_{t}-K_{t} H_{s}=0$. So we can give the following theorem.

Theorem 4.3. The surface $\psi(s, t)$ is a Weingarten surface.

Now assume that there exist real numbers $a, b, c \in \mathbb{R} \backslash\{0\}$ such that the linear combination $a K+b H=c$ is satisfied.

$$
a K+b H-c=\frac{b-2 c r+2\left(a-c r^{2}+b r\right) \sinh t-2\left(a-c r^{2}+b r\right) \kappa \cosh t}{2 r(1+r \sinh t-r \kappa \cosh t)}=0
$$

which implies that $b=2 c r$ and $a+c r^{2}=0$. So we can give the following theorem.

Theorem 4.4. Let $K$ and $H$ be the Gauss curvature and mean curvature of the surface $\psi(s, t)$. Then there exists the following relation between $K$ and $H$ :

$$
-r^{2} K+2 r H=1
$$

where $r$ is a positive real number.

From above theorem, we get the following corollary. 
Corollary 4.1. The surface $\psi(s, t)$ is a linear Weingarten surface.

Definition 4.1. The parallel surface of the surface $X(s, t)$ defined by

$$
X^{*}(s, t)=X(s, t)+\mu U(s, t)
$$

where

$$
U(s, t)=\frac{X_{s} \times X_{t}}{\left\|X_{s} \times X_{t}\right\|}
$$

is the unit normal vector of the surface $X(s, t)$ and $\mu \in \mathbb{R}$.

Now we will define the parallel surface $\psi^{*}(s, t)$ of the surface $\psi(s, t)$ as follows

$$
\begin{aligned}
\psi^{*}(s, t) & =\psi(s, t)+\mu e_{3} \\
& =(1+(r-\mu) \sinh t) x(s)+((r-\mu) \cosh t) y(s)
\end{aligned}
$$

From (4.4), we find

$$
\begin{aligned}
\psi_{s}^{*} & =(1+(r-\mu) \sinh t-(r-\mu) \kappa \cosh t) \alpha, \\
\psi_{t}^{*} & =((r-\mu) \cosh t) x-((r-\mu) \sinh t) y .
\end{aligned}
$$

We can find the components of first fundemental form as follows

$$
\begin{aligned}
& g_{11}^{*}=\left\langle\psi_{s}^{*}, \psi_{s}^{*}\right\rangle=(1+(r-\mu) \sinh t-(r-\mu) \kappa \cosh t)^{2}, \\
& g_{12}^{*}=\left\langle\psi_{s}^{*}, \psi_{t}^{*}\right\rangle=0, \quad g_{22}=\left\langle\psi_{t}, \psi_{t}\right\rangle=-(r-\mu)^{2} .
\end{aligned}
$$

Then $g_{11}^{*} g_{22}^{*}-\left(g_{12}^{*}\right)^{2}=(r-\mu)^{2}(1+(r-\mu) \sinh t-(r-\mu) \kappa \cosh t)^{2}$. We assume that $r-\mu>0$ and $1+$ $(r-\mu) \sinh t-(r-\mu) \kappa \cosh t>0$ for the regularity of the surface $\psi^{*}(s, t)$.

Now we will give an orthonormal basis on $\psi^{*}(s, t)$.

$$
\begin{aligned}
& e_{1}^{*}=\frac{1}{\left\|\psi_{s}^{*}\right\|} \psi_{s}^{*}=\alpha, \\
& e_{2}^{*}=\frac{1}{\left\|\psi_{t}^{*}\right\|} \psi_{t}^{*}=(\cosh t) x+(\sinh t) y,
\end{aligned}
$$

where $\left\{e_{1}^{*}, e_{2}^{*}\right\}$ is an orthonormal frame field on $\psi^{*}(s, t)$. Set

$$
e_{3}^{*}=-(\sinh t) x-(\cosh t) y,
$$

where $e_{3}^{*}$ is a normal vector field to $\psi^{*}(s, t) \cdot\left\{e_{1}^{*}, e_{2}^{*}, e_{3}^{*}\right\}$ is an orthonormal basis on $\psi^{*}(s, t)$. Then we obtain

$$
\begin{aligned}
D_{e_{1}^{*}} e_{1}^{*} & =\frac{1}{1+(r-\mu) \sinh t-(r-\mu) \kappa \cosh t}(x+\kappa y), \\
D_{e_{1}^{*}} e_{2}^{*} & =\frac{\cosh t-\kappa \sinh t}{1+(r-\mu) \sinh t-(r-\mu) \kappa \cosh t} \alpha, \\
D_{e_{2}^{*}} e_{2}^{*} & =\frac{1}{(r-\mu)}((\sinh t) x+(\cosh t) y) .
\end{aligned}
$$

The components of the second fundamental form $h^{*}$ are calculated as follows

$$
\begin{aligned}
& h_{11}^{*}=\left\langle D_{e_{1}^{*}} e_{1}^{*}, e_{3}^{*}\right\rangle=\frac{\sinh t-\kappa \cosh t}{1+(r-\mu) \sinh t-(r-\mu) \kappa \cosh t}, \\
& h_{12}^{*}=\left\langle D_{e_{1}^{*}} e_{2}^{*}, e_{3}^{*}\right\rangle=0 \text { and } h_{22}=\left\langle D_{e_{2}^{*}}^{*} e_{2}^{*}, e_{3}^{*}\right\rangle=-\frac{1}{(r-\mu)} .
\end{aligned}
$$

Similarly we can find the following results.

Theorem 4.5. The mean curvature $H^{*}$ of $\psi^{*}(s, t)$ is obtained as

$$
H^{*}=\frac{1-2(r-\mu) \kappa \cosh t+2(r-\mu) \sinh t}{2(r-\mu)(1+(r-\mu) \sinh t-(r-\mu) \kappa \cosh t)} .
$$


Theorem 4.6. The Gauss curvature $K^{*}$ of $\psi^{*}(s, t)$ is obtained as

$$
K^{*}=\frac{\sinh t-\kappa \cosh t}{(r-\mu)(1+(r-\mu) \sinh t-(r-\mu) \kappa \cosh t)} .
$$

Theorem 4.7. The surface $\psi^{*}(s, t)$ is a Weingarten surface.

Theorem 4.8. Let $K^{*}$ and $H^{*}$ be the Gauss curvature and mean curvature of the surface $\psi^{*}(s, t)$. Then there exists the following relation between $K^{*}$ and $H^{*}$ :

$$
-(r-\mu)^{2} K^{*}+2(r-\mu) H^{*}=1
$$

where $r$ is a positive real number and $\mu$ is a real number.

From above theorem, we get the following corollary.

Corollary 4.2. The surface $\psi^{*}(s, t)$ is a linear Weingarten surface.

Definition 4.2. Let $x_{1}(\bar{s})$ be the associated curve of $x(s)$ such that $x_{1}(\bar{s})=y(s)$ where there exists a diffeomorfism $\bar{s}=f_{1}(s)$. In this paper, we will call $x_{1}(\bar{s})=y(s)$ as the first associated curve of the hyperbolic curve $x(s)$.

Let $x_{2}\left(s^{*}\right)=\alpha(s)$ where there exists a diffeomorfism $s^{*}=f_{2}(s)$ Then we will call $x_{2}\left(s^{*}\right)=\alpha(s)$ as the second associated curve of the hyperbolic curve $x(s)$.

Definition 4.3. Let $x_{1}(\bar{s})$ be the first associated curve of the hyperbolic curve $x(s)$ in $H^{2}, \psi(s, t)$ and $\psi_{1}(\bar{s}, t)$ be canal surfaces (or tubular surfaces) whose center curves are $x(s)$ and $x_{1}(\bar{s})$, respectively. Then $\psi_{1}(\bar{s}, t)$ is called as "the first associated canal surface (or the first associated tubular surface)" of $\psi(s, t)$.

Similarly, let $x_{2}\left(s^{*}\right)$ be the second associated curve of the hyperbolic curve $x(s)$ in $H^{2}, \psi(s, t)$ and $\psi_{2}\left(s^{*}, t\right)$ be canal surfaces (or tubular surfaces) whose center curves are $x(s)$ and $x_{2}\left(s^{*}\right)$, respectively. Then $\psi_{2}\left(s^{*}, t\right)$ is called as "the second associated canal surface (or the second associated tubular surface)" of $\psi(s, t)$.

4.1.1. The first associated curve and the first associated tubular surface Firstly we consider the first associated curve and the first associated tubular surface of the hyperbolic curve $x(s)$. So we can give the following corollary.

Corollary 4.3. Let $x_{1}(\bar{s})$ be the first associated curve of the hyperbolic curve $x(s)$ in $H^{2}$ with the hyperbolic frame $\{x(s), \alpha(s), y(s)\}$ such that $x_{1}(\bar{s})=y(s)$ where there exists a diffeomorfism $\bar{s}=f_{1}(s)$. Then we have

$$
x_{1}=y, \quad \alpha_{1}=-\varepsilon_{1} \alpha, \quad y_{1}=-\varepsilon_{1} x, \quad \kappa_{1}=\frac{\varepsilon_{1}}{\kappa}, \quad \frac{d f_{1}}{d s}=\varepsilon_{1} \kappa,
$$

and

$$
\frac{d x_{1}}{d \bar{s}}=\alpha_{1}, \quad \frac{d \alpha_{1}}{d \bar{s}}=-x_{1}+\kappa_{1} y_{1}, \quad \frac{d y_{1}}{d \bar{s}}=\kappa_{1} \alpha_{1}
$$

where $\varepsilon_{1}=\operatorname{sgn}(\kappa),\left\{x_{1}(\bar{s}), \alpha_{1}(\bar{s}), y_{1}(\bar{s})\right\}$ is the spherical frame, $\kappa_{1}(\bar{s})$ is the spherical curvature of $x_{1}(\bar{s})$ and

$$
\left\langle x_{1}(\bar{s}), x_{1}(\bar{s})\right\rangle=\left\langle\alpha_{1}(\bar{s}), \alpha_{1}(\bar{s})\right\rangle=1, \quad\left\langle y_{1}(\bar{s}), y_{1}(\bar{s})\right\rangle=-1 .
$$

Theorem 4.9. Let $x_{1}(\bar{s})$ be the first associated curve of the hyperbolic curve $x(s)$ in $H^{2}$ with the hyperbolic frame $\{x(s), \alpha(s), y(s)\}$ such that $x_{1}(\bar{s})=y(s)$. Also let $x_{1}(\bar{s})$ be the center curve of a canal surface

(i) obtained from the sphere $S^{2}(r)$. Then the parametrization of the canal surface can be as following

$$
\begin{aligned}
M_{1}(\bar{s}, t)= & \left(1+m_{1} r(\bar{s}) \sqrt{1-r_{s}^{2}(\bar{s})} \cosh t\right) x_{1}(\bar{s})-r(\bar{s}) r_{s}(\bar{s}) \alpha_{1}(\bar{s}) \\
& +\left(m_{2} r(\bar{s}) \sqrt{1-r_{s}^{2}(\bar{s})} \sinh t\right) y_{1}(\bar{s})
\end{aligned}
$$

and the parametrization of the tubular surface can be as following

$$
M_{1}(\bar{s}, t)=\left(1+m_{1} r \cosh t\right) x_{1}(\bar{s})+\left(m_{2} r \sinh t\right) y_{1}(\bar{s})
$$


(ii) obtained from the hyperbolic space $H^{2}(r)$. Then the parametrization of the canal surface can be as following

$$
\begin{aligned}
M_{2}(\bar{s}, t)= & \left(1+m_{1} r(\bar{s}) \sqrt{1+r_{s}^{2}(\bar{s})} \sinh t\right) x_{1}(\bar{s})+r(\bar{s}) r_{s}(\bar{s}) \alpha_{1}(\bar{s}) \\
& +\left(m_{2} r(\bar{s}) \sqrt{1+r_{s}^{2}(\bar{s})} \cosh t\right) y_{1}(\bar{s})
\end{aligned}
$$

and the parametrization of the tubular surface can be as following

$$
M_{2}(\bar{s}, t)=\left(1+m_{1} r \sinh t\right) x_{1}(\bar{s})+\left(m_{2} r \cosh t\right) y_{1}(\bar{s})
$$

(iii) obtained from the ligthlike cone $Q^{2}$. Then the parametrization of the canal surface(tubular surface) can be as following

$$
M_{3}(\bar{s}, t)=(1+a(\bar{s}, t)) x_{1}(\bar{s})+m_{1} a(\bar{s}, t) y_{1}(\bar{s})
$$

where $m_{1}, m_{2} \in\{-1,1\}$ and $a(\bar{s}, t)$ is a function of $\bar{s}$ and $t$, but not only $\bar{s}$.

Firstly we consider the tubular surface $M_{1}(\bar{s}, t)$ for $m_{1}=m_{2}=1$ as follows

$$
\begin{aligned}
M_{1}(\bar{s}, t) & =(1+r \cosh t) x_{1}(\bar{s})+(r \sinh t) y_{1}(\bar{s}) \\
& =-\varepsilon_{1}(r \sinh t) x(s)+(1+r \cosh t) y(s) .
\end{aligned}
$$

From (4.5), we have

$$
\begin{aligned}
\left(M_{1}\right)_{\bar{s}} & =\frac{-\varepsilon_{1}\left(\varepsilon_{1} r \sinh t+\kappa(1+r \cosh t)\right)}{\kappa} \alpha \\
\left(M_{1}\right)_{t} & =-\left(\varepsilon_{1} r \cosh t\right) x+(r \sinh t) y
\end{aligned}
$$

which implies that

$$
\begin{aligned}
& \left\langle\left(M_{1}\right)_{\bar{s}},\left(M_{1}\right)_{\bar{s}}\right\rangle=\frac{\left(\varepsilon_{1} r \sinh t+\kappa(1+r \cosh t)\right)^{2}}{\kappa^{2}}, \\
& \left\langle\left(M_{1}\right)_{\bar{s}},\left(M_{1}\right)_{t}\right\rangle=0, \quad\left\langle\left(M_{1}\right)_{t},\left(M_{1}\right)_{t}\right\rangle=-r^{2}
\end{aligned}
$$

Then

$$
\left\langle\left(M_{1}\right)_{\bar{s}},\left(M_{1}\right)_{\bar{s}}\right\rangle\left\langle\left(M_{1}\right)_{t},\left(M_{1}\right)_{t}\right\rangle-\left\langle\left(M_{1}\right)_{\bar{s}},\left(M_{1}\right)_{t}\right\rangle^{2}=-r^{2} \frac{\left(\varepsilon_{1} r \sinh t+\kappa(1+r \cosh t)\right)^{2}}{\kappa^{2}} .
$$

Theorem 4.10. Let $M_{1}(\bar{s}, t)$ be the first associated tubular surfaces of $M(s, t)$, which is obtained by $S_{1}^{2}(r)$. Then $M_{1}(\bar{s}, t)$ has a singular point at $M\left(s_{0}, t_{0}\right)$ if and only if

$$
\varepsilon_{1} r \sinh t_{0}+\kappa\left(s_{0}\right)\left(1+r \cosh t_{0}\right)=0 .
$$

Now we assume that $\varepsilon_{1} r \sinh t_{0}+\kappa\left(s_{0}\right)\left(1+r \cosh t_{0}\right) \neq 0$ for all $(t, s)$. Then we will give an orthonormal basis on $M_{1}(\bar{s}, t)$.

$$
\begin{aligned}
\bar{e}_{1} & =\frac{1}{\left\|\left(M_{1}\right)_{\bar{s}}\right\|}\left(M_{1}\right)_{\bar{s}}=-\varepsilon_{2} \alpha, \\
\bar{e}_{2} & =\frac{1}{\left\|\left(M_{1}\right)_{t}\right\|}\left(M_{1}\right)_{t}=-\left(\varepsilon_{1} \cosh t\right) x+(\sinh t) y,
\end{aligned}
$$

where $\varepsilon_{2}=\operatorname{sgn}\left(\varepsilon_{1} r \sinh t+\kappa(1+r \cosh t)\right)$ and $\left\{\bar{e}_{1}, \bar{e}_{2}\right\}$ is an orthonormal frame field on $M_{1}(\bar{s}, t)$. Set

$$
\bar{e}_{3}=-(\sinh t) x-\left(\varepsilon_{1} \cosh t\right) y,
$$

where $\bar{e}_{3}$ is a normal vector field to $M_{1}(\bar{s}, t) \cdot\left\{\bar{e}_{1}, \bar{e}_{2}, \bar{e}_{3}\right\}$ is an orthonormal basis on $M_{1}(\bar{s}, t)$. Then we obtain

$$
\begin{aligned}
D_{\bar{e}_{1}} \bar{e}_{1} & =\frac{-1}{\varepsilon_{1} r \sinh t+\kappa(1+r \cosh t)}(x+\kappa y), \\
D_{\bar{e}_{1}} \bar{e}_{2} & =\frac{-\varepsilon_{2}\left(\varepsilon_{1} \cosh t+\kappa \sinh t\right)}{\varepsilon_{1} r \sinh t+\kappa(1+r \cosh t)} \alpha, \\
D_{\bar{e}_{2}} \bar{e}_{2} & =\frac{1}{r}\left(-\left(\varepsilon_{1} \sinh t\right) x+(\cosh t) y\right) .
\end{aligned}
$$


The components of the second fundamental form $\bar{h}$ are calculated as follows

$$
\begin{aligned}
& \bar{h}_{11}=\left\langle D_{\bar{e}_{1}} \bar{e}_{1}, \bar{e}_{3}\right\rangle=\frac{\varepsilon_{1} \kappa \cosh t-\sinh t}{\varepsilon_{1} r \sinh t+\kappa(1+r \cosh t)}, \\
& \bar{h}_{12}=\left\langle D_{\bar{e}_{1}} \bar{e}_{2}, \bar{e}_{3}\right\rangle=0 \text { and } \bar{h}_{22}=\left\langle D_{\bar{e}_{2}} \bar{e}_{2}, \bar{e}_{3}\right\rangle=\frac{\varepsilon_{1}}{r} .
\end{aligned}
$$

Theorem 4.11. The mean curvature $H_{1}$ of $M_{1}(\bar{s}, t)$ is obtained as

$$
H_{1}=\frac{1}{2}\left(\bar{h}_{11}-\bar{h}_{22}\right)=\frac{-\varepsilon_{1}\left(2 \varepsilon_{1} r \sinh t+\kappa(1+2 r \cosh t)\right)}{2 r\left(\varepsilon_{1} r \sinh t+\kappa(1+r \cosh t)\right)} .
$$

Theorem 4.12. The Gauss curvature $K_{1}$ of $M_{1}(\bar{s}, t)$ is obtained as

$$
K_{1}=-\left(\bar{h}_{11} \bar{h}_{22}-\left(\bar{h}_{12}\right)^{2}\right)=\frac{\varepsilon_{1}\left(\sinh t+\varepsilon_{1} \kappa \cosh t\right)}{r\left(\varepsilon_{1} r \sinh t+\kappa(1+r \cosh t)\right)} .
$$

Thus it can be easily seen that $\Psi\left(K_{1}, H_{1}\right)=\left(K_{1}\right)_{\bar{s}}\left(H_{1}\right)_{t}-\left(K_{1}\right)_{t}\left(H_{1}\right)_{\bar{s}}=0$. So we can give the following theorems.

Theorem 4.13. The surface $M_{1}(\bar{s}, t)$ is a Weingarten surface.

Theorem 4.14. Let $K_{1}$ and $H_{1}$ be the Gauss curvature and mean curvature of the surface $M_{1}(\bar{s}, t)$. Then there exists the following relation between $K_{1}$ and $H_{1}$ :

$$
-r^{2} K_{1}-2 \varepsilon_{1} r H_{1}=1
$$

where $r$ is a positive real number.

From above theorem, we get the following corollary.

Corollary 4.4. The surface $M_{1}(\bar{s}, t)$ is a linear Weingarten surface.

Now we consider the tubular surface $M_{2}(\bar{s}, t)$ for $m_{1}=m_{2}=1$ as follows

$$
\begin{aligned}
M_{2}(\bar{s}, t) & =(1+r \sinh t) x_{1}(\bar{s})+(r \cosh t) y_{1}(\bar{s}) \\
& =\left(-\varepsilon_{1} r \cosh t\right) x(s)+(1+r \sinh t) y(s) .
\end{aligned}
$$

Similarly we can find the followings.

Theorem 4.15. Let $M_{2}(\bar{s}, t)$ be the first associated tubular surfaces of $M(s, t)$, which is obtained by $H^{2}(r)$. Then $M_{2}(\bar{s}, t)$ has a singular point at $M\left(s_{0}, t_{0}\right)$ if and only if

$$
\varepsilon_{1} r \cosh t_{0}+\kappa\left(s_{0}\right)\left(1+r \sinh t_{0}\right)=0 .
$$

Theorem 4.16. The mean curvature $\mathrm{H}_{2}$ of $M_{2}(\bar{s}, t)$ is obtained as

$$
H_{2}=\frac{\varepsilon_{1}\left(2 \varepsilon_{1} r \cosh t+\kappa(1+2 r \sinh t)\right)}{2 r\left(\varepsilon_{1} r \cosh t+\kappa(1+r \sinh t)\right)} .
$$

Theorem 4.17. The Gauss curvature $K_{2}$ of $M_{2}(\bar{s}, t)$ is obtained as

$$
K_{2}=\frac{-\varepsilon_{1}\left(\cosh t+\varepsilon_{1} \kappa \sinh t\right)}{r\left(\varepsilon_{1} r \cosh t+\kappa(1+r \sinh t)\right)} .
$$

Thus it can be easily seen that $\Psi\left(K_{2}, H_{2}\right)=\left(K_{2}\right)_{\bar{s}}\left(H_{2}\right)_{t}-\left(K_{2}\right)_{t}\left(H_{2}\right)_{\bar{s}}=0$. So we can give the following theorems.

Theorem 4.18. The surface $M_{2}(\bar{s}, t)$ is a Weingarten surface.

Theorem 4.19. Let $K_{2}$ and $H_{2}$ be the Gauss curvature and mean curvature of the surface $M_{2}(\bar{s}, t)$. Then there exists the following relation between $K_{1}$ and $H_{1}$ :

$$
r^{2} K_{2}+2 \varepsilon_{1} r H_{2}=1
$$

where $r$ is a positive real number.

From above theorem, we get the following corollary.

Corollary 4.5. The surface $M_{2}(\bar{s}, t)$ is a linear Weingarten surface.

Remark 4.1. Let $M_{3}(\bar{s}, t)$ be the first associated tubular surfaces of $M(s, t)$, which is obtained by $Q^{2}$. Then $M_{3}(\bar{s}, t)$ is a degenerate surface. 
4.1.2. The second associated curve and the second associated tubular surface Now, we consider the second associated curve and the second associated tubular surface of the hyperbolic curve $x(s)$. So we can give the following corollaries.

Corollary 4.6. Let $x_{2}\left(s^{*}\right)$ be the second associated curve of the hyperbolic curve $x(s)$ with $\kappa^{2}>1$ in $H^{2}$ with the hyperbolic frame $\{x(s), \alpha(s), y(s)\}$ such that $x_{2}\left(s^{*}\right)=\alpha(s)$ where there exists a diffeomorfism $s^{*}=f_{2}(s)$. Then we have

$$
\begin{aligned}
x_{2} & =\alpha, \quad \alpha_{2}=\frac{1}{\sqrt{\kappa^{2}-1}}(x+\kappa y), \quad y_{2}=\frac{1}{\sqrt{\kappa^{2}-1}}(-\kappa x-y), \\
\kappa_{2} & =\frac{\kappa^{\prime}}{\left(\kappa^{2}-1\right)^{3 / 2}}, \quad \frac{d f_{2}}{d s}=\sqrt{\kappa^{2}-1}
\end{aligned}
$$

and

$$
\frac{d x_{2}}{d s^{*}}=\alpha_{2}, \quad \frac{d \alpha_{2}}{d s^{*}}=-x_{2}+\kappa_{2} y_{2}, \quad \frac{d y_{2}}{d s^{*}}=\kappa_{2} \alpha_{2}
$$

where $\left\{x_{2}\left(s^{*}\right), \alpha_{2}\left(s^{*}\right), y_{2}\left(s^{*}\right)\right\}$ is the spherical frame, $\kappa_{2}\left(s^{*}\right)$ is the spherical curvature of $x_{2}\left(s^{*}\right)$ and

$$
\left\langle x_{2}\left(s^{*}\right), x_{2}\left(s^{*}\right)\right\rangle=\left\langle\alpha_{2}\left(s^{*}\right), \alpha_{2}\left(s^{*}\right)\right\rangle=1, \quad\left\langle y_{2}\left(s^{*}\right), y_{2}\left(s^{*}\right)\right\rangle=-1 .
$$

Theorem 4.20. Let $x_{2}\left(s^{*}\right)$ be the second associated curve of the hyperbolic curve $x(s)$ with $\kappa^{2}>1$ in $H^{2}$ with the hyperbolic frame $\{x(s), \alpha(s), y(s)\}$ such that $x_{2}\left(s^{*}\right)=\alpha(s)$. Also let $x_{2}\left(s^{*}\right)$ be the center curve of a canal surface $(i)$ obtained from the sphere $S^{2}(r)$. Then the parametrization of the canal surface can be as following

$$
\begin{aligned}
M_{4}\left(s^{*}, t\right)= & \left(1+m_{1} r\left(s^{*}\right) \sqrt{1-r_{s}^{2}\left(s^{*}\right)} \cosh t\right) x_{2}\left(s^{*}\right)-r\left(s^{*}\right) r_{s}\left(s^{*}\right) \alpha_{2}\left(s^{*}\right) \\
& +\left(m_{2} r\left(s^{*}\right) \sqrt{1-r_{s}^{2}\left(s^{*}\right)} \sinh t\right) y_{2}\left(s^{*}\right)
\end{aligned}
$$

and the parametrization of the tubular surface can be as following

$$
M_{4}\left(s^{*}, t\right)=\left(1+m_{1} r \cosh t\right) x_{2}\left(s^{*}\right)+\left(m_{2} r \sinh t\right) y_{2}\left(s^{*}\right)
$$

(ii) obtained from the hyperbolic space $H^{2}(r)$. Then the parametrization of the canal surface can be as following

$$
\begin{aligned}
M_{5}\left(s^{*}, t\right)= & \left(1+m_{1} r\left(s^{*}\right) \sqrt{1+r_{s}^{2}\left(s^{*}\right)} \sinh t\right) x_{2}\left(s^{*}\right)+r\left(s^{*}\right) r_{s}\left(s^{*}\right) \alpha_{2}\left(s^{*}\right) \\
& +\left(m_{2} r\left(s^{*}\right) \sqrt{1+r_{s}^{2}\left(s^{*}\right)} \cosh t\right) y_{2}\left(s^{*}\right)
\end{aligned}
$$

and the parametrization of the tubular surface can be as following

$$
M_{5}\left(s^{*}, t\right)=\left(1+m_{1} r \sinh t\right) x_{2}\left(s^{*}\right)+\left(m_{2} r \cosh t\right) y_{2}\left(s^{*}\right)
$$

(iii) obtained from the ligthlike cone $Q^{2}$. Then the parametrization of the canal surface(tubular surface) can be as following

$$
M_{6}\left(s^{*}, t\right)=\left(1+a\left(s^{*}, t\right)\right) x_{2}\left(s^{*}\right)+m_{1} a\left(s^{*}, t\right) y_{2}\left(s^{*}\right)
$$

where $m_{1}, m_{2} \in\{-1,1\}$ and $a\left(s^{*}, t\right)$ is a function of $s^{*}$ and $t$, but not only $s^{*}$.

Corollary 4.7. Let $x_{2}\left(s^{*}\right)$ be the second associated curve of the hyperbolic curve $x(s)$ with $\kappa^{2}<1$ in $H^{2}$ with the hyperbolic frame $\{x(s), \alpha(s), y(s)\}$ such that $x_{2}\left(s^{*}\right)=\alpha(s)$ where there exists a diffeomorfism $s^{*}=f_{2}(s)$. Then we have

$$
\begin{aligned}
x_{2} & =\alpha, \quad \alpha_{2}=\frac{1}{\sqrt{1-\kappa^{2}}}(x+\kappa y), \quad y_{2}=\frac{1}{\sqrt{1-\kappa^{2}}}(-\kappa x-y) \\
\kappa_{2} & =\frac{\kappa^{\prime}}{\left(1-\kappa^{2}\right)^{3 / 2}}, \quad \frac{d f_{2}}{d s}=\sqrt{1-\kappa^{2}}
\end{aligned}
$$

and

$$
\frac{d x_{2}}{d s^{*}}=\alpha_{2}, \quad \frac{d \alpha_{2}}{d s^{*}}=x_{2}-\kappa_{2} y_{2}, \quad \frac{d y_{2}}{d s^{*}}=-\kappa_{2} \alpha_{2}
$$

where $\left\{x_{2}\left(s^{*}\right), \alpha_{2}\left(s^{*}\right), y_{2}\left(s^{*}\right)\right\}$ is the spherical frame, $\kappa_{2}\left(s^{*}\right)$ is the spherical curvature of $x_{2}\left(s^{*}\right)$ and

$$
\left\langle x_{2}\left(s^{*}\right), x_{2}\left(s^{*}\right)\right\rangle=\left\langle y_{2}\left(s^{*}\right), y_{2}\left(s^{*}\right)\right\rangle=1, \quad\left\langle\alpha_{2}\left(s^{*}\right), \alpha_{2}\left(s^{*}\right)\right\rangle=-1 .
$$


Theorem 4.21. Let $x_{2}\left(s^{*}\right)$ be the second associated curve of the hyperbolic curve $x(s)$ with $\kappa^{2}<1$ in $H^{2}$ with the hyperbolic frame $\{x(s), \alpha(s), y(s)\}$ such that $x_{2}\left(s^{*}\right)=\alpha(s)$. Also let $x_{2}\left(s^{*}\right)$ be the center curve of a canal surface (i) obtained from the sphere $S^{2}(r)$. Then the parametrization of the canal surface can be as following

$$
\begin{aligned}
M_{7}\left(s^{*}, t\right)= & \left(1+m_{1} r\left(s^{*}\right) \sqrt{1+r_{s}^{2}\left(s^{*}\right)} \cos t\right) x_{2}\left(s^{*}\right)+r\left(s^{*}\right) r_{s}\left(s^{*}\right) \alpha_{2}\left(s^{*}\right) \\
& +\left(m_{2} r\left(s^{*}\right) \sqrt{1+r_{s}^{2}\left(s^{*}\right)} \sin t\right) y_{2}\left(s^{*}\right)
\end{aligned}
$$

and the parametrization of the tubular surface can be as following

$$
M_{7}\left(s^{*}, t\right)=\left(1+m_{1} r \cos t\right) x_{2}\left(s^{*}\right)+\left(m_{2} r \sin t\right) y_{2}\left(s^{*}\right)
$$

(ii) obtained from the hyperbolic space $H^{2}(r)$. Then the parametrization of the canal surface can be as following

$$
\begin{aligned}
M_{8}\left(s^{*}, t\right)= & \left(1+m_{1} r\left(s^{*}\right) \sqrt{r_{s}^{2}\left(s^{*}\right)-1} \cos t\right) x_{2}\left(s^{*}\right)-r\left(s^{*}\right) r_{s}\left(s^{*}\right) \alpha_{2}\left(s^{*}\right) \\
& +\left(m_{2} r\left(s^{*}\right) \sqrt{r_{s}^{2}\left(s^{*}\right)-1} \sin t\right) y_{2}\left(s^{*}\right),
\end{aligned}
$$

where $m_{1}, m_{2} \in\{-1,1\}$.

Now, we consider the second associated tubular surface $M_{4}\left(s^{*}, t\right)$ of $M(s, t)$. Assume that $\kappa(s)=\kappa$ (constant). Let $M_{4}\left(s^{*}, t\right)$ be the second associated tubular surface of $M(s, t)$. Then we can write

$$
\begin{aligned}
M_{4}\left(s^{*}, t\right) & =(1+r \cosh t) x_{2}\left(s^{*}\right)+(r \sinh t) y_{2}\left(s^{*}\right) \\
& =-\frac{\kappa r \sinh t}{\sqrt{\kappa^{2}-1}} x(s)+(1+r \cosh t) \alpha(s)+\frac{r \sinh t}{\sqrt{\kappa^{2}-1}} y(s) .
\end{aligned}
$$

From (4.8), we have

$$
\begin{aligned}
\left(M_{4}\right)_{s^{*}} & =\frac{1+r \cosh t}{\sqrt{\kappa^{2}-1}}(-x+\kappa y), \\
\left(M_{4}\right)_{t} & =\frac{-\kappa r \cosh t}{\sqrt{\kappa^{2}-1}} x+(r \sinh t) \alpha-\frac{r \cosh t}{\sqrt{\kappa^{2}-1}} y,
\end{aligned}
$$

which implies that

$$
\left\langle\left(M_{4}\right)_{s^{*}},\left(M_{4}\right)_{s^{*}}\right\rangle=(1+r \cosh t)^{2}, \quad\left\langle\left(M_{4}\right)_{s^{*}},\left(M_{4}\right)_{t}\right\rangle=0, \quad\left\langle\left(M_{4}\right)_{t},\left(M_{4}\right)_{t}\right\rangle=-r^{2} .
$$

Then

$$
\left\langle\left(M_{4}\right)_{s^{*}},\left(M_{4}\right)_{s^{*}}\right\rangle\left\langle\left(M_{4}\right)_{t},\left(M_{4}\right)_{t}\right\rangle-\left\langle\left(M_{4}\right)_{s^{*}},\left(M_{4}\right)_{t}\right\rangle^{2}=-r^{2}(1+r \cosh t)^{2} .
$$

Theorem 4.22. Let $M_{4}\left(s^{*}, t\right)$ be the second associated tubular surfaces of $M(s, t)$. Then $M_{4}\left(s^{*}, t\right)$ has a singular point at $M\left(s, t_{0}\right)$ if and only if $1+r \cosh t_{0}=0$.

Now we assume that $1+r \cosh t \neq 0$ for all $(t, s)$. Then we will give an orthonormal basis on $M_{4}\left(s^{*}, t\right)$.

$$
\begin{aligned}
e_{1}^{*} & =\frac{1}{\left\|\left(M_{4}\right)_{s^{*}}\right\|}\left(M_{4}\right)_{s^{*}}=\frac{\varepsilon_{2}}{\sqrt{\kappa^{2}-1}}(x+\kappa y), \\
e_{2}^{*} & =\frac{1}{\left\|\left(M_{4}\right)_{t}\right\|}\left(M_{4}\right)_{t}=-\frac{\kappa \cosh t}{\sqrt{\kappa^{2}-1}} x+(\sinh t) \alpha-\frac{\cosh t}{\sqrt{\kappa^{2}-1}} y,
\end{aligned}
$$

where $\varepsilon_{2}=\operatorname{sgn}(1+r \cosh t)$ and $\left\{e_{1}^{*}, e_{2}^{*}\right\}$ is an orthonormal frame field on $M_{4}\left(s^{*}, t\right)$. Set

$$
e_{3}^{*}=\frac{\varepsilon_{2} \kappa \sinh t}{\sqrt{\kappa^{2}-1}} x-\left(\varepsilon_{2} \cosh t\right) \alpha+\frac{\varepsilon_{2} \sinh t}{\sqrt{\kappa^{2}-1}} y,
$$

where $e_{3}^{*}$ is a normal vector field to $M_{4}\left(s^{*}, t\right) \cdot\left\{e_{1}^{*}, e_{2}^{*}, e_{3}^{*}\right\}$ is an orthonormal basis on $M_{4}\left(s^{*}, t\right)$. Then we obtain

$$
\begin{aligned}
D_{e_{1}^{*}} e_{1}^{*} & =\frac{1}{1+r \cosh t} \alpha \\
D_{e_{1}^{*}} e_{2}^{*} & =\frac{\varepsilon_{2} \sinh t}{(1+r \cosh t) \sqrt{\kappa^{2}-1}}(x+\kappa y), \\
D_{e_{2}^{*}} e_{2}^{*} & =-\frac{\kappa \sinh t}{r \sqrt{\kappa^{2}-1}} x+\frac{\cosh t}{r} \alpha-\frac{\sinh t}{r \sqrt{\kappa^{2}-1}} y .
\end{aligned}
$$


The components of the second fundamental form $h^{*}$ are calculated as follows

$$
\begin{aligned}
& h_{11}^{*}=\left\langle D_{e_{1}^{*}} e_{1}^{*}, e_{3}^{*}\right\rangle=\frac{-\varepsilon_{2} \cosh t}{1+r \cosh t}, \\
& h_{12}^{*}=\left\langle D_{e_{1}^{*}} e_{2}^{*}, e_{3}^{*}\right\rangle=0 \text { and } h_{22}^{*}=\left\langle D_{e_{2}^{*}} e_{2}^{*}, e_{3}^{*}\right\rangle=-\frac{\varepsilon_{2}}{r} .
\end{aligned}
$$

Theorem 4.23. The mean curvature $H_{2}$ of $M_{4}\left(s^{*}, t\right)$ is obtained as

$$
H_{2}=\frac{\varepsilon_{2}}{2 r(1+r \cosh t)} .
$$

Theorem 4.24. The Gauss curvature $K_{2}$ of $M_{4}\left(s^{*}, t\right)$ is obtained as

$$
K_{2}=\frac{-\cosh t}{r(1+r \cosh t)} .
$$

From (4.9) and (4.10), we have

$$
\left(K_{2}\right)_{s^{*}}=0, \quad\left(K_{2}\right)_{t}=\frac{-\sinh t}{r(1+r \cosh t)^{2}}
$$

and

$$
\left(H_{2}\right)_{s^{*}}=0, \quad\left(H_{2}\right)_{t}=\frac{-\sinh t}{2(1+r \cosh t)^{2}} .
$$

Thus it can be easily seen that $\Psi\left(K_{2}, H_{2}\right)=0$. So we can give the following theorem.

Theorem 4.25. The surface $M_{4}\left(s^{*}, t\right)$ is a Weingarten surface.

Also we can give the following theorem.

Theorem 4.26. Let $K_{2}$ and $H_{2}$ be the Gauss curvature and mean curvature of the surface $M_{4}\left(s^{*}, t\right)$. Then there exists the following relation between $K_{2}$ and $H_{2}$ :

$$
-r^{2} K_{2}+2 \varepsilon_{2} r H_{2}=1
$$

where $r$ is a positive real number.

From above theorem, we get the following corollary.

Corollary 4.8. The surface $M_{4}\left(s^{*}, t\right)$ is a linear Weingarten surface.

The second Gaussian curvature $K_{I I}$ of the surface $M_{4}\left(s^{*}, t\right)$ is obtained that

$$
K_{I I}=\frac{1+2 r^{2}+6 r \cosh t+2 r^{2} \cosh 2 t+\operatorname{sech}^{2} t}{4 \varepsilon_{2} r(1+r \cosh t)^{2}} .
$$

Then it can be easily seen that $\Psi\left(K_{I I}, H_{2}\right)=0$ and $\Psi\left(K_{I I}, K_{2}\right)=0$. So we can give the following theorem.

Theorem 4.27. The surface $M_{4}\left(s^{*}, t\right)$ is a $(X, Y)$-Weingarten surface where $(X, Y) \in\left\{\left(K_{2}, K_{I I}\right),\left(H_{2}, K_{I I}\right)\right\}$.

Remark 4.2. Similar properties can be easily obtained for the tubular surfaces $M_{5}\left(s^{*}, t\right)$ and $M_{7}\left(s^{*}, t\right)$.

Remark 4.3. The tubular surface $M_{6}\left(s^{*}, t\right)$ is a degenerate surface.

\subsection{Tubular surface obtained from the sphere $H^{2}(r)$}

This subsection is omitted from the paper since the properties can be obtained similarly to previous subsection. 


\section{References}

[1] Chen B.-Y.: Geometry of Submanifolds. Dekker, New York (1973).

[2] Gök İ.: Quaternionic approach of canal surfaces constructed by some new ideas. Adv. Appl. Clifford Algebras. 27 (2), 1175-1190 (2017).

[3] İlarslan K. and Boyacıŏglu Ö.: Position vectors of a spacelike W-curve in Minkowski space $\mathbb{E}_{1}^{3}$. Bulletin of the Korean Mathematical Society. 44 (3), 429-438 (2007).

[4] İlarslan K. and Boyacığlu Ö.: Position vectors of a timelike and a null helix in Minkowski 3-space. Chaos, Solitons \& Fractals, 38 (5), 1383-1389 (2008).

[5] Karacan M. K., Es H. and Yayl1, Y.: Singular points of the Tubular Surfaces in Minkowski 3-space. Sarajevo Journal of Mathematics. 14, 73-82 (2006).

[6] Karacan M. K., Yoon D. W. and Tuncer Y.: Tubular Surfaces of Weingarten Types in Minkowski 3-space. Gen. Math. Notes. 22, 44-56 (2014).

[7] Karacan M. K. and Bukcu B.: An alternative moving frame for a tubular surface around a spacelike curve with a spacelike normal in Minkowski 3-space. Rendiconti del Circolo Matematico di Palermo.57, 193-201 (2008).

[8] Karacan M. K. and Tuncer Y.: Tubular Surfaces of Weingarten types in Galilean and Pseudo-Galilean. Bulletin of Mathematical Analysis and Applications. 5, 87-100 (2013).

[9] Kocakuşaklı E., Tuncer O. O., Gök İ. and YaylıY.: A new representation of canal surfaces with split quaternions in Minkowski 3-space. Adv. Appl. Clifford Algebras. 27 (2), 1387-1409 (2017).

[10] Liu H.: Curves in three dimensional Riemannian space forms. Results. Math. 66, 469-480 (2014).

[11] Petrović-Torgašev M. and Šućurović E.: W-curves in Minkowski space-time. Novi Sad J. Math. 32 (2), 55-65 (2002).

[12] Ro J. S. and Yoon D. W.: Tubes of Weingarten types in a Euclidean 3-space. Journal of the Chungcheong mathematical society. 22 (3), $359-366$ (2009).

[13] Uçum A., İlarslan, K.: New types of canal surfaces in Minkowski 3-space. Adv. Appl. Clifford Algebras. 26, 449-468 (2016).

[14] Yoon D. W.: On non-developable ruled surfaces in Euclidean 3-spaces. Indian Journal of Pure and Applied Mathematics. 38, 281-290 (2007).

\section{Affiliations}

\section{ALI UÇUM}

AdDrEss: Kırıkkale University, Faculty of Sciences and Arts, Department of Mathematics, Kirıkkale-Turkey.

E-MAIL: aliucum05@gmail.com

ORCID ID: 0000-0003-0172-1531 